\title{
AURIFEUILLIAN FACTORIZATIONS AND THE PERIOD OF THE BELL NUMBERS MODULO A PRIME
}

\author{
SAMUEL S. WAGSTAFF, JR.
}

\begin{abstract}
We show that the minimum period modulo $p$ of the Bell exponential integers is $\left(p^{p}-1\right) /(p-1)$ for all primes $p<102$ and several larger $p$. Our proof of this result requires the prime factorization of these periods. For some primes $p$ the factoring is aided by an algebraic formula called an Aurifeuillian factorization. We explain how the coefficients of the factors in these formulas may be computed.
\end{abstract}

\section{INTRODUCTION}

The first-order Bell exponential integers $B(n)$ may be defined by the generating function

$$
e^{e^{x}-1}=\sum_{n=0}^{\infty} B(n) \frac{x^{n}}{n !}
$$

These integers appear in many combinatorial problems. For example, $B(n)$ is the number of ways a product of $n$ different primes may be factored. See [6] and its references for more background.

Williams [13] proved that for each prime $p$ the sequence $\{B(n) \bmod p ; n=$ $0,1, \ldots\}$ is periodic and that the minimum period divides

$$
N_{p}=\frac{p^{p}-1}{p-1}
$$

He showed that the minimum period is precisely $N_{p}$ for $p=2,3$ and 5 . Levine and Dalton [6] showed that the minimum period is exactly $N_{p}$ for $p=7,11,13$ and 17. They also investigated the period for the other primes $<50$. We show that the minimum period is exactly $N_{p}$ for each prime $<102$ and for several larger primes. Our technique is the same one used by Levine and Dalton. We show that the period is not $N_{p} / q$ for any prime factor $q$ of $N_{p}$. We were able to extend their work so far because of great advances in integer factoring methods since 1962.

In the next two sections we describe our attempts to factor $N_{p}$ for primes $p<180$. The final section explains how we investigated the period of $\{B(n) \bmod p\}$.

Received by the editor August 24, 1993 and, in revised form, January 26, 1995.

1991 Mathematics Subject Classification. Primary 11-04, 11B73; Secondary 11Y05, 12-04, 12E10, $12 \mathrm{Y} 05$

Key words and phrases. Bell numbers, period modulo $p$, integer factorization, Lucas' identities, Aurifeuillian factorization.

Some of the computing reported in this work was performed on a MasPar computer at Purdue University which was supported in part by NSF Infrastructure Grant CDA-9015696. 


\section{Factorization of $N_{p}$}

As we tried to factor $N_{p}$ for the odd primes $p<180$, we also tried to factor the important related numbers $K_{p}=\left(p^{p}+1\right) /(p+1)$ for the same primes $p$. It is well known that all prime factors of $N_{p}$ and $K_{p}$ have the form $2 k p+1$, where $k$ is a positive integer. After just a little trial division we used the Elliptic Curve Method [5]. We used the Quadratic Sieve Method [9] to factor the occasional integer of modest size which did not succumb to the Elliptic Curve Method. Before we did any of this work, however, we used the fact that for each odd prime $p$, one of $N_{p}, K_{p}$ admits an algebraic factorization into two nearly equal factors. In fact, if $p$ is squarefree, then the numbers $\left(p^{h p}-1\right) /(p-1)$ when $p \equiv 1(\bmod 4)$ and $\left(p^{h p}+1\right) /(p+1)$ when $p \equiv 2$ or $3(\bmod 4)$ have algebraic factorizations for all odd $h$. Although we describe these factorizations in general in Theorem 2, in this paper we use only the case $h=1$ and $p$ prime. The algebraic factorizations are called Aurifeuillian because some of these formulas were discovered by Aurifeuille (see page 276 of [7]).

The known factors of $N_{p}$ and $K_{p}$ are given in Tables 1 and 2. The notations $P x x$ and $C x x$ denote prime and composite numbers of $x x$ digits. An $L$ or $M$ following $p$ refers to the Aurifeuillian factor of Theorem 2 below.

Levine and Dalton [6] copied some factors from the table in Cunningham [4] including the erroneous "factor" 6709 of $N_{43}$, and found more factors by trial division. But they did not use the Aurifeuillian factorizations from [4]. If they had, they could have finished factoring $N_{29}$ and probably also $N_{37}$.

TABLE 1: Factorization of $N_{p}=\left(p^{p}-1\right) /(p-1)$ for primes $p<180$

$\begin{array}{ll}p & \text { Known prime factors of } N_{p} \\ 3 & 13 \\ 5 L & 11 \\ 5 M & 71 \\ 7 & 29.4733 \\ 11 & 15797.1806113 \\ 13 L & 1803647 \\ 13 M & 53.264031 \\ 17 L & 2699538733 \\ 17 M & 10949.1749233 \\ 19 & 109912203092239643840221 \\ 23 & 461.1289 .831603031789 .1920647391913 \\ 29 L & 84449.2428577 .549334763 \\ 29 M & 59.16763 .14111459 .58320973 \\ 31 & 568972471024107865287021434301977158534824481 \\ 37 L & 149.41903425553544839998158239 \\ 37 M & 1999.7993 .16651 .17317 .10192715656759 \\ 41 L & 1752341.20567159 .1876859311090803007 \\ 41 M & 83.5926187589691497537793497756719 \\ 43 & 173.120401 . P 62 \\ 47 & 1693.255742492896763511474638530188876017 . P 39\end{array}$


TABLE 1 (continued)

\begin{tabular}{|c|c|}
\hline$p$ & Known prime factors of $N_{p}$ \\
\hline $53 L$ & 107.16505521259654533 .143470720478589313288313473 \\
\hline $53 M$ & 141829.13033960579631324880455449881408994392143 \\
\hline 59 & 709.141579233.P92 \\
\hline $61 L$ & 977.343625872243632312073 .398853286456071792609917995907 \\
\hline $61 M$ & 1000403244183535565720394723140528028235711874491322863 \\
\hline 67 & $\begin{array}{l}269.4021 .730837 .10960933 . \\
\quad .1514954885096604023562287915730049 . P 69\end{array}$ \\
\hline 71 & 105649.3388409395214741.17882954877203881.P93 \\
\hline $73 L$ & 1414741.1295720382587.1192167517020392933.P31 \\
\hline $73 M$ & 293.439.25239167.56377463.3611379501352361.P32 \\
\hline 79 & $\begin{array}{c}317.1558537597 .171355071830508389477 . \\
.54493132908043378263202913 . P 91\end{array}$ \\
\hline 83 & 2657.11155201.1008505707601323349156769489.P120 \\
\hline $89 L$ & 179.8009862103557709.5964844210432006407836201.P43 \\
\hline $89 M$ & 37307598912253490893302199133.P58 \\
\hline $97 L$ & P95 \\
\hline $97 M$ & 389.363751.684640163.11943728733741294764390602153.P51 \\
\hline $101 L$ & 1213.9931988588681.102208068907493.393101595766008847.P53 \\
\hline $101 M$ & $607.5657 .157561 . P 89$ \\
\hline 103 & 1237.16706917226363953216841.C180 \\
\hline 107 & 137122213.10508824813.C197 \\
\hline $109 L$ & $2617 . C 107$ \\
\hline $109 M$ & 6196098743139082891438631.P86 \\
\hline $113 L$ & 3391.8363.785192800256197898644431714786031.P75 \\
\hline $113 M$ & $\begin{array}{l}227.34314816732569 . \\
\quad .70739255769077616674066085318030811655932920203 . P 53\end{array}$ \\
\hline 127 & $509.22861 .1320675600886906675359917 . C 234$ \\
\hline 131 & 1049.1742643541410742623061.C251 \\
\hline $137 L$ & $54142883557383383180139791 . C 120$ \\
\hline $137 M$ & $\begin{array}{l}1097.124123 .1918644449 .12779722229 .574894288613 . \\
.271329112787027 .1759429467460935879916775610180659 . P 59\end{array}$ \\
\hline 139 & $557.119833345601 . C 282$ \\
\hline $149 L$ & 1193.C158 \\
\hline $149 M$ & $51784951.450090559 .465814231 . C 137$ \\
\hline 151 & $2417.15101 .1234577 . C 314$ \\
\hline $157 L$ & $1356984109417 . C 159$ \\
\hline $157 M$ & 86351.P167 \\
\hline 163 & $\begin{array}{l}653.2609 .41729 .31943437 .3727539197017 .391683908074297 . \\
\quad .8224734227858383253 . P 294\end{array}$ \\
\hline 167 & 16033.1001953110409.669806250678629514045626189.P326 \\
\hline $173 L$ & 347.685081.P184 \\
\hline $173 M$ & 161297590410850151.P176 \\
\hline 179 & $C 402$ \\
\hline
\end{tabular}


TABLE 2. Factorization of $K_{p}=\left(p^{p}+1\right) /(p+1)$ for primes $p<180$

$\begin{array}{ll}p & \text { Known prime factors of } K_{p} \\ 3 & 7 \\ 5 & 521 \\ 7 L & 113 \\ 7 M & 911 \\ 11 L & 58367 \\ 11 M & 23.89 .199 \\ 13 & 13417.20333 .79301 \\ 17 & 45957792327018709121 \\ 19 L & 108301.1049219 \\ 19 M & 870542161121 \\ 23 L & 47.139 .1013 .52626071 \\ 23 M & 1641281.1522029233 \\ 29 & 233.6864997 .9487923853 .5639663878716545087233 \\ 31 L & 1613.145577 .35789156484227 \\ 31 M & 373.62869 .2706690202468649 \\ 37 & 593.134135213 .4356032201 .6190006021 . P 27 \\ 41 & 18041.20396681 . P 53 \\ 43 L & 947.6709 .1140834804168935454622067377 \\ 43 M & 1291.86689 .485926008972226664331036683 \\ 47 L & 65519.10519189757 .60963223421 .2506611914519 \\ 47 M & 659.15511 .21179047 .3543413924249049822089893 \\ 53 & 991313.2644277 .5324593 .14443842647093 .19604216783737 . P 45 \\ 59 L & 27759619.6806872605199 .4393717192308664068865841443741 \\ 59 M & 4466419.11821911653180627 .114888627555970745944996436263 \\ 61 & 2441.1191941 .9229762307875553 .560622532089629629 . \\ & \quad .28523716939675891427869 . P 42 \\ 67 L & P 60 \\ 67 M & 141907.4002983 .5759607944561 . P 37 \\ 71 L & 4872163.7270495362831024364754355287 . P 30 \\ 71 M & 17467.59743093 . P 54 \\ 73 & 4596369165585291112352829637852339157090144708807832677 . P 80 \\ 79 L & P 74 \\ 79 M & 34919.188021 .45780868646549 . P 51 \\ 83 L & 499.9463 . P 72 \\ 83 M & 167.997 .17929 .472168956426245957 .860785395874331487431 . P 32 \\ 89 & 169573582127857.11188457211131513436831539501 . P 130 \\ 97 & 1553.1631871607681574053 . C 170 \\ 101 & 10741549365517.266345719946724536329 . C 167 \\ 103 L & 2267.18541 .237313 .43577750158649183 . \\ & .1133217861836283429782583969130809253 . P 37 \\ 103 M & 1031.692779 .36733862315539624797022993014846462017 . P 57 \\ 107 L & 1061227.46242619 .304535269 . \\ 107 M & \quad .3211610951880144183669785219693807857 . P 49 \\ 109 & 1236165024989.10341388749337445617033 . P 186 \\ & \\ & \\ & \end{array}$


TABLE 2 (continued)

\begin{tabular}{|c|c|}
\hline$p$ & Known prime factors of $K_{p}$ \\
\hline 113 & 2713.108637220969.76199628846557168921.C196 \\
\hline $127 \mathrm{~L}$ & 921259.1525238541798558622809202213.P99 \\
\hline $127 M$ & 5932933.26759010325255571935109471.P101 \\
\hline $131 L$ & $\begin{array}{l}263.8123 .23581 .128119 .509192023 .5434194401 .118531075451349793 . \\
\quad .2274827737024993390020446837627 . P 56\end{array}$ \\
\hline $131 M$ & $\begin{array}{l}3407.16003103839 .8425818148421874530481343817 . \\
\quad .405970466949758035428707456821 . P 67\end{array}$ \\
\hline 137 & 136453.164095915779277.C272 \\
\hline $139 L$ & $25577 . C 144$ \\
\hline $139 M$ & 21374190911672122661.1977185134537749396577.P108 \\
\hline 149 & $\begin{array}{l}3513009953.4907466108140806981 .915115125488764974144697 . \\
\quad .2809439870825424714368565313 . C 242\end{array}$ \\
\hline $151 L$ & $\begin{array}{l}53593223.20110202953 .322631539451020618739 . \\
.21410447638232281941934857667 . P 97\end{array}$ \\
\hline $151 M$ & 7853.C160 \\
\hline 157 & P343 \\
\hline $163 L$ & P179 \\
\hline $163 M$ & 6521.185821.2272547.21163569551.C154 \\
\hline $167 L$ & 3760684691.14974117420259.C162 \\
\hline $167 M$ & 8017.3295913.465247639.4386303138831827.C151 \\
\hline 173 & C385 \\
\hline $179 L$ & 359.1433.909679.113069992151013739136227.P166 \\
\hline $179 M$ & 1597039.5864420639771327037769.C173 \\
\hline
\end{tabular}

\section{Aurifeuillian Factorizations}

For integers $n>0$ let $\Phi_{n}(x)$ denote the cyclotomic polynomial

$$
\Phi_{n}(x)=\prod_{\substack{j=1 \\(j, n)=1}}^{n}\left(x-\zeta_{n}^{j}\right),
$$

where $\zeta_{n}$ is a primitive $n$th root of unity. It is well known that $x^{n}-1=\prod_{d \mid n} \Phi_{d}(x)$. If $p$ is an odd prime, then $\left(x^{p}-1\right) /(x-1)=\Phi_{p}(x)$ and $\left(x^{p}+1\right) /(x+1)=\Phi_{2 p}(x)$. Thus $N_{p}=\Phi_{p}(p)$ and $K_{p}=\Phi_{2 p}(p)$. Although $\Phi_{n}(x)$ is irreducible over the integers, it may be reducible over certain quadratic fields. Theorem 1 sets the stage for some factorizations of this type. The first two parts of Theorem 1 were proved by Lucas [8]. Schinzel [11] gave a modern proof of the entire theorem. Our Theorem 1 is the case $m=n$ of Theorem 1 of [11]. Let $(m \mid n)$ be the Jacobi symbol. For $\sqrt{c}$ we make the convention $\sqrt{c} \geq 0$ if $c \geq 0$ and $\sqrt{c}=i \sqrt{-c}$ if $c<0$.

Theorem 1. Let $n>1$ be a squarefree integer. Then there exist polynomials $P_{n}(x)$ and $Q_{n}(x)$ with integer coefficients such that

$$
\Phi_{n}(x)=P_{n}^{2}(x)-(-1 \mid n) n x Q_{n}^{2}(x) \quad \text { and } \quad \Phi_{2 n}(x)=P_{n}^{2}(-x)+(-1 \mid n) n x Q_{n}^{2}(-x)
$$


when $n$ is odd, and

$$
\Phi_{2 n}(x)=P_{n}^{2}(x)-n x Q_{n}^{2}(x)
$$

when $n$ is even. These polynomials can be computed from the formulas

$$
\begin{gathered}
P_{n}\left(x^{2}\right)-\sqrt{(-1 \mid n) n} x Q_{n}\left(x^{2}\right)=\prod_{s}\left(x-\zeta_{n}^{s}\right) \prod_{t}\left(x+\zeta_{n}^{t}\right), \\
P_{n}\left(-x^{2}\right)-i \sqrt{(-1 \mid n) n} x Q_{n}\left(-x^{2}\right)=\prod_{s}\left(x+i \zeta_{n}^{s}\right) \prod_{t}\left(x-i \zeta_{n}^{t}\right),
\end{gathered}
$$

where the products are over $0<s<n, 0<t<n,(s t, n)=1,(s \mid n)=1,(t \mid n)=-1$ when $n$ is odd, and from the formula

$$
P_{n}\left(x^{2}\right)-\sqrt{n} x Q_{n}\left(x^{2}\right)=\prod_{s}\left(x-\zeta_{4 n}^{s}\right),
$$

where the product is over $0<s<4 n,(s, 4 n)=1,(n \mid s)=1$ when $n$ is even.

It is easy to modify Theorem 1 to use only real numbers. Theorem 2 does this and also restricts the identities to cases when they produce interesting Aurifeuillian factorizations, that is, when the cyclotomic polynomial is expressed as the difference of two squares. Let $\phi(n)$ denote Euler's totient function.

Theorem 2. Let $n>1$ be an odd squarefree integer. Then there exist polynomials $C_{n}(x)$ and $D_{n}(x)$ with integer coefficients and degrees $\phi(n) / 2$ and $\phi(n) / 2-1$, respectively, with the following properties. Let $h$ be an odd positive integer. If $n \equiv 1$ $(\bmod 4)$, then

$$
\Phi_{n}\left(n^{h}\right)=\left(C_{n}\left(n^{h}\right)-n^{(h+1) / 2} D_{n}\left(n^{h}\right)\right)\left(C_{n}\left(n^{h}\right)+n^{(h+1) / 2} D_{n}\left(n^{h}\right)\right),
$$

and if $n \equiv 3(\bmod 4)$, then

$$
\Phi_{2 n}\left(n^{h}\right)=\left(C_{n}\left(n^{h}\right)-n^{(h+1) / 2} D_{n}\left(n^{h}\right)\right)\left(C_{n}\left(n^{h}\right)+n^{(h+1) / 2} D_{n}\left(n^{h}\right)\right) .
$$

The coefficients of $C_{n}(x)$ and $D_{n}(x)$ may be computed from the identity

$$
C_{n}\left(x^{2}\right)-\sqrt{n} x D_{n}\left(x^{2}\right)=\prod_{\substack{s=1 \\(s, n)=1}}^{(n-1) / 2}\left(x^{2}-2(s \mid n) f_{n}(s) x+1\right),
$$

where $f_{n}(s)=\cos \frac{2 \pi s}{n}$ if $n \equiv 1(\bmod 4)$ and $f_{n}(s)=\sin \frac{2 \pi s}{n}$ if $n \equiv 3(\bmod 4)$.

Let $n$ be an even squarefree positive integer. Then there exist polynomials $C_{n}(x)$ and $D_{n}(x)$ with integer coefficients and degrees $\phi(n)$ and $\phi(n)-1$, respectively, so that (1) holds when $h$ is an odd positive integer. The coefficients of $C_{n}(x)$ and $D_{n}(x)$ may be computed from the identity

$$
C_{n}\left(x^{2}\right)-\sqrt{n} x D_{n}\left(x^{2}\right)=\prod_{\substack{s=1 \\(s, n)=1}}^{2 n}\left(x^{2}-(1+(n \mid s)) \cos \frac{\pi s}{2 n} x+1\right) .
$$

Proof. Let $n \equiv 1(\bmod 4)$. Then $(-1 \mid n)=1$. By Theorem $1, \Phi_{n}(x)=P_{n}^{2}(x)-$ $n x Q_{n}^{2}(x)$, where

$$
P_{n}\left(x^{2}\right)-\sqrt{n} x Q_{n}\left(x^{2}\right)=\prod_{\substack{s=1 \\(s, n)=1}}^{n-1}\left(x-(s \mid n) \zeta_{n}^{s}\right)
$$


In the product combine the factors with $s$ and $n-s$. Note that $(s, n)=1$ if and only if $(n-s, n)=1$. Also $(n-s \mid n)=(s \mid n)$ and $\zeta_{n}^{s}+\zeta_{n}^{n-s}=2 \cos \frac{2 \pi s}{n}$. The product of the two factors is $x^{2}-2(s \mid n) 2 \cos \frac{2 \pi s}{n} x+1$. Writing $C_{n}(x)=P_{n}(x)$, $D_{n}(x)=Q_{n}(x)$ and $x=n^{h}$ gives the result. There are $\phi(n) / 2$ quadratic factors in the product in (2), so the degree of the polynomial in $(2)$ is $\phi(n)$. Since this polynomial is $C_{n}\left(x^{2}\right)-\sqrt{n} x D_{n}\left(x^{2}\right)$, the degree of $C_{n}$ is $\phi(n) / 2$ and the degree of $D_{n}$ is $\phi(n) / 2-1$.

Now let $n \equiv 3(\bmod 4)$. Then $(-1 \mid n)=-1$. By Theorem $1, \Phi_{2 n}(x)=P_{n}^{2}(-x)-$ $n x Q_{n}^{2}(-x)$, where

$$
P_{n}\left(-x^{2}\right)-i \sqrt{n} x Q_{n}\left(-x^{2}\right)=\prod_{\substack{s=1 \\(s, n)=1}}^{n-1}\left(x+i(s \mid n) \zeta_{n}^{s}\right) .
$$

In the product combine the factors with $s$ and $n-s$. Note that $(s, n)=1$ if and only if $(n-s, n)=1$. Also $(n-s \mid n)=-(s \mid n)$ and $\zeta_{n}^{s}-\zeta_{n}^{n-s}=2 i \sin \frac{2 \pi s}{n}$. The product of the two factors is $x^{2}-2(s \mid n) 2 \sin \frac{2 \pi s}{n} x+1$. Writing $C_{n}(x)=P_{n}(-x)$, $D_{n}(x)=Q_{n}(-x)$ and $x=n^{h}$ gives the result.

Now suppose $n$ is even. Then $n \equiv 2(\bmod 4)$ because $n$ is squarefree. By Theorem $1, \Phi_{2 n}(x)=P_{n}^{2}(x)-n x Q_{n}^{2}(x)$, where

$$
P_{n}\left(x^{2}\right)-\sqrt{n} x Q_{n}\left(x^{2}\right)=\prod_{\substack{s=1 \\(s, 4 n)=1 \\(n \mid s)=1}}^{4 n}\left(x-\zeta_{4 n}^{s}\right) .
$$

In the product combine the factors with $s$ and $4 n-s$. Note that $(s, 4 n)=1$ if and only if $(4 n-s, 4 n)=1$. Also $(n \mid 4 n-s)=(n \mid s)$ and $\zeta_{4 n}^{s}+\zeta_{4 n}^{4 n-s}=2 \cos \frac{2 \pi s}{4 n}$. The product of the two factors is $x^{2}-(1+(n \mid s)) \cos \frac{\pi s}{2 n} x+1$. Since $(n, s)=1$, the factor $(1+(n \mid s))$ is 2 when $(n \mid s)=1$ and is 0 when $(n \mid s)=-1$. Writing $C_{n}(x)=P_{n}(x)$, $D_{n}(x)=Q_{n}(x)$ and $x=n^{h}$ gives the result and proves Theorem 2 .

The two factors of $\Phi_{n}(n)$ or $\Phi_{2 n}(n)$ in Theorem 2 are denoted $n L$ and $n M$ in Tables 1 and 2. A table of coefficients of $C_{n}(x)$ and $D_{n}(x)$ for $n<120$ may be found in Table 34 on page $453 \mathrm{ff}$. of Riesel [10].

Ordinary 64-bit double-precision floating-point arithmetic permits the correct calculation in a fraction of a second of these coefficients for odd $n<180$. The program was tested by comparing the product of $n L$ and $n M$, computed from $C_{n}(n)$ and $D_{n}(n)$, with $N_{n}$ or $K_{n}$, computed independently.

Brent [2] gives an algorithm for computing the coefficients of $C_{n}(x)$ and $D_{n}(x)$ which uses integer arithmetic throughout.

\section{The period of $\{B(n) \bmod p\}$}

When $p$ is prime, this period is known to be a divisor of $N_{p}$. To test whether the period divides some factor $N$ of $N_{p}$, it is enough to compare $B(N+i) \bmod p$ with $B(i) \bmod p$ for $1 \leq i \leq p$. Only these $p$ pairs need to be compared because the congruence

$$
B(n+p) \equiv B(n)+B(n+1) \quad(\bmod p)
$$

of Touchard [12] shows that any $p$ consecutive values of $B(n) \bmod p$ determine the sequence after that point. For each prime divisor $q$ of $N_{p}$ listed in Table 1, the 
test just described was performed for $N=N_{p} / q$. When we could not factor $N_{p}$ completely, we performed the test also for $N_{p}$ divided by its remaining composite cofactor $q$ (two of them for $N_{149}$ ). In every case the outcome of the test was that the period did not divide $N_{p} / q$. We also performed the test with $N=N_{p}$ to check the program. The not unexpected outcome was that $N_{p}$ is a period. Finally, we tested some $N_{p}$ with $p>180$ to see whether the period might be slightly smaller than $N_{p}$. Specifically, for each prime $p$ in $180<p<1100$ we computed all primes $q<2^{31}$ dividing $N_{p}$ and tested $N_{p} / q$ for being a period. It never was a period. Thus, we have proved the following result.

Theorem 3. The minimum period of the sequence $\{B(n) \bmod p\}$ is $N_{p}$ when $p$ is a prime $<102$ and also when $p=113,163,167$ or 173 . For the remaining primes $p<180$, no proper divisor of $N_{p}$ whose codivisor appears in Table 1 is a period of the sequence. Furthermore, for each prime $p<1100$, no proper divisor of $N_{p}$ whose codivisor has only prime factors $<2^{31}$ is a period of the sequence.

Based on the evidence provided by Theorem 3, we conjecture that the minimum period of the sequence $\{B(n) \bmod p\}$ is $N_{p}$ for every prime $p$.

It remains to explain how we computed $B(N) \bmod p$ when $p$ is a prime $<1100$ and $N$ is large; some $N$ have thousands of decimal digits. First of all, we computed $b_{i}=B(i) \bmod p$ for $0 \leq i \leq p$ using the formula $B(n+1)=\sum_{j=0}^{n}\left(\begin{array}{l}n \\ j\end{array}\right) B(j)$ of Cesaro [3] (see also Becker and Browne [1]). That is, we used this algorithm:

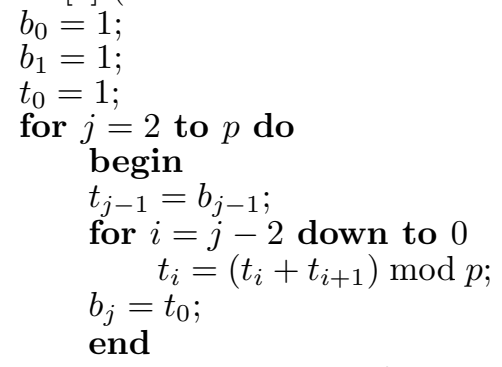

This algorithm takes $\mathrm{O}\left(N^{2}\right)$ operations to compute $B(N) \bmod p$, so it is too slow to use for large $N$. To compute $B(N) \bmod p$ for large $N$ we use the congruence $B\left(n+p^{m}\right) \equiv B(n+1)+m B(n)(\bmod p)$ of Touchard [12], which generalizes $(3)$. We write $N$ in radix $p$ as $N=\sum_{i=0}^{e} a_{i} p^{i}$, where $0 \leq a_{i}<p$ and $a_{e} \neq 0$. Starting from the block $b_{i}=B(i) \bmod p, 0 \leq i \leq p$, we use the digits $a_{i}$ to compute other blocks of length $p+1$ of values of $B(i) \bmod p$. The algorithm, which runs in $\mathrm{O}\left(p^{2} \log N\right)$ steps, is:

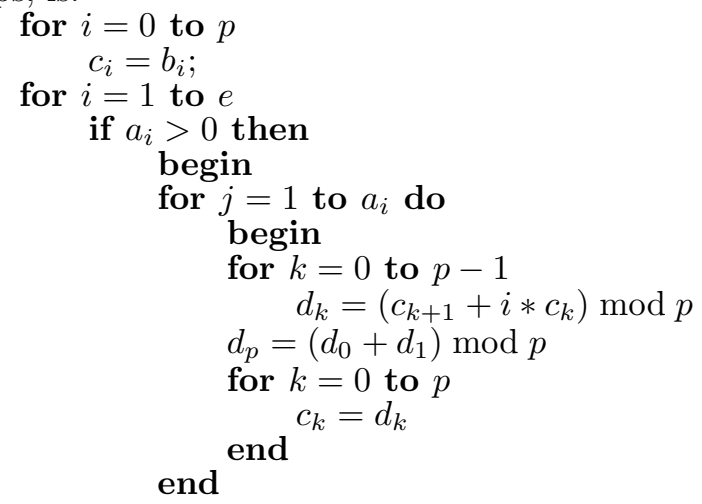


At this point, $c_{a_{0}}$ is $B(N) \bmod p$. Use (3) to shift the window to $B(N+i) \bmod p$ for $0 \leq i \leq p$. Then compare $B(N+i) \bmod p$ with $b_{i}$ for $0 \leq i \leq p$ to decide whether $N$ is a period. For every proper divisor $N$ of $N_{p}$ that we examined, either $B(N) \bmod p \neq b_{0}$ or $B(N+1) \bmod p \neq b_{1}$.

\section{ACKNOWLEDGEMENTS}

I thank John W. Wrench, Jr. for suggesting this research and R. P. Brent for sending me a preprint of [2]. I am indebted to Harvey Cohn and Hugh Edgar for valuable discussions of this research. I discovered many factors using an ECM program written by P. L. Montgomery. He computed Table 1 independently and sent me several factors which I had missed. I am grateful to Marije Huizing of the Centrum voor Wiskunde en Informatica in Amsterdam for factoring $K_{73}$ by the number field sieve. I thank A. K. Lenstra and B. Dixon for letting me use their ECM program for the MasPar computer. It found several factors.

\section{REFERENCES}

1. H. W. Becker and D. H. Browne, Problem E461 and solution, Amer. Math. Monthly 48 (1941), 701-703.

2. Richard P. Brent, On computing factors of cyclotomic polynomials, Math. Comp. 61 (1993), 131-149. MR 93m:11131

3. E. Cesaro, Sur une équation aux différences mêlées, Nouvelles Annales de Math. (3) 4 (1885), $36-40$.

4. A. J. C. Cunningham, Factorisation of $N=y^{y} \mp 1$ and $x^{x y} \mp y^{x y}$, Messenger of Math. (2) 45 (1915), 49-75.

5. H. W. Lenstra, Jr., Factoring integers with elliptic curves, Ann. of Math. (2) 126 (1987), 649-673. MR 89g:11125

6. Jack Levine and R. E. Dalton, Minimum periods, modulo p, of first-order Bell exponential integers, Math. Comp. 16 (1962), 416-423. MR 26:6111

7. E. Lucas, Théorèms d'arithmétique, Atti R. Accad. Sci. Torino 13 (1877-78), 271-284.

8. 783-789.

9. Carl Pomerance, The quadratic sieve factoring algorithm, Advances in Cryptology, Proceedings of EUROCRYPT 84 (T. Beth, N. Cot, and I. Ingemarsson, eds.), Springer-Verlag Lecture Notes in Comput. Sci., vol. 209, 1985, pp. 169-182. MR 86m:94003

10. Hans Riesel, Prime numbers and computer methods for factorization, Birkhäuser, Boston, 1985. MR 88k: 11002

11. A. Schinzel, On the primitive prime factors of $a^{n}-b^{n}$, Proc. Cambridge Philos. Soc. 58 (1962), 555-562. MR 26:1280

12. J. Touchard, Propriétés arithmétiques de certains nombres recurrents, Ann. Soc. Sci. Bruxelles 53A (1933), 21-31.

13. G. T. Williams, Numbers generated by the function $e^{e^{x}-1}$, Amer. Math. Monthly 52 (1945), 323-327. MR 7:47e

Department of Computer Sciences, Purdue University, West Lafayette, Indiana 47907

E-mail address: ssw@cs.purdue.edu 